\title{
Front Matter: Volume 11274
}

, "Front Matter: Volume 11274," Proc. SPIE 11274, Physics and Simulation of Optoelectronic Devices XXVIII, 1127401 (21 April 2020); doi:

$10.1117 / 12.2570291$

SPIE. Event: SPIE OPTO, 2020, San Francisco, California, United States 
PROCEEDINGS OF SPIE

\title{
Physics and Simulation of Optoelectronic Devices XXVIII
}

\author{
Bernd Witzigmann \\ Marek Osiński \\ Yasuhiko Arakawa \\ Editors
}

3-6 February 2020

San Francisco, California, United States

Sponsored and Published by

SPIE

Volume 11274 
The papers in this volume were part of the technical conference cited on the cover and title page. Papers were selected and subject to review by the editors and conference program committee. Some conference presentations may not be available for publication. Additional papers and presentation recordings may be available online in the SPIE Digital Library at SPIEDigitalLibrary.org.

The papers reflect the work and thoughts of the authors and are published herein as submitted. The publisher is not responsible for the validity of the information or for any outcomes resulting from reliance thereon.

Please use the following format to cite material from these proceedings:

Author(s), "Title of Paper," in Physics and Simulation of Optoelectronic Devices XXVIII, edited by Bernd Witzigmann, Marek Osiński, Yasuhiko Arakawa, Proceedings of SPIE Vol. 11274 (SPIE, Bellingham, WA, 2020) Seven-digit Article CID Number.

ISSN: 0277-786X

ISSN: 1996-756X (electronic)

ISBN: 9781510633117

ISBN: 9781510633124 (electronic)

Published by

SPIE

P.O. Box 10, Bellingham, Washington 98227-0010 USA

Telephone +1 3606763290 (Pacific Time) · Fax + 13606471445

SPIE.org

Copyright @ 2020, Society of Photo-Optical Instrumentation Engineers.

Copying of material in this book for internal or personal use, or for the internal or personal use of specific clients, beyond the fair use provisions granted by the U.S. Copyright Law is authorized by SPIE subject to payment of copying fees. The Transactional Reporting Service base fee for this volume is $\$ 21.00$ per article (or portion thereof), which should be paid directly to the Copyright Clearance Center (CCC), 222 Rosewood Drive, Danvers, MA 01923. Payment may also be made electronically through CCC Online at copyright.com. Other copying for republication, resale, advertising or promotion, or any form of systematic or multiple reproduction of any material in this book is prohibited except with permission in writing from the publisher. The CCC fee code is 0277$786 \times / 20 / \$ 21.00$.

Printed in the United States of America by Curran Associates, Inc., under license from SPIE.

Publication of record for individual papers is online in the SPIE Digital Library.

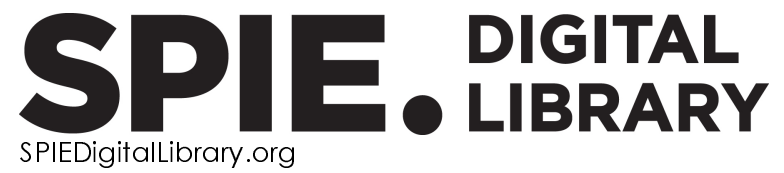

Paper Numbering: Proceedings of SPIE follow an e-First publication model. A unique citation identifier (CID) number is assigned to each article at the time of publication. Utilization of CIDs allows articles to be fully citable as soon as they are published online, and connects the same identifier to all online and print versions of the publication. SPIE uses a seven-digit CID article numbering system structured as follows:

- The first five digits correspond to the SPIE volume number.

- The last two digits indicate publication order within the volume using a Base 36 numbering system employing both numerals and letters. These two-number sets start with $00,01,02,03,04$, 05, 06, 07, 08, 09, OA, OB ... 0Z, followed by 10-1Z, 20-2Z, etc. The CID Number appears on each page of the manuscript. 


\section{Contents}

$\begin{array}{ll}\text { vii } & \text { Authors } \\ \text { ix } & \text { Conference Committee }\end{array}$

ELECTROMAGNETICS/PLASMONICS I

$1127403 \quad$ Broadband infrared metamaterial absorber with raindrop-shaped submicron-scale disk array [1 1 $1274-2]$

$1127404 \quad$ Linear Schrödinger equation for front-induced transitions close to the band edge [1 1274-3]

1127405 Graphene-based c-shaped metasurface broadband solar absorber [1 1274-4]

\section{ACTIVE MATERIALS FOR OPTOELECTRONICS}

$1127408 \quad$ InAs/InAsSb type 2 superlattices band parameters determination via magnetoabsorption and k.p modeling [1 1274-7]

$11274 \mathrm{OA} \quad$ Role of point-defect charging dynamics in quantum-well magneto-transport [1 $1274-9]$

\section{MINISYMPOSIUM ON NEUROMORPHIC COMPUTING WITH OPTICAL DEVICES}

11274 OD Integrated photonic delay-lasers for reservoir computing (Invited Paper) [1 1274-11]

11274 OF Automatic classification of video using a scalable photonic neuro-inspired architecture (Invited Paper) [1 1274-12]

SEMICONDUCTOR LASERS: NUMERICAL MODELING

$11274 \mathrm{Ol} \quad$ Traveling wave model-based analysis of tapered broad-area lasers [1 1274-15]

$112740 \mathrm{~J} \quad$ Impact of dislocations in monolithic III-V lasers on silicon: a theoretical approach [1 1274-16]

11274 OK Energy balance model of high-power semiconductor lasers at high-pumping current [1 1 $1274-17]$

$11274 \mathrm{OL} \quad$ Turn on transient in a long cavity laser [1 1274-18] 
SIGNAL ENCRYPTION AND NONLINEAR OPTICAL SYSTEMS

$112740 \mathrm{OM}$ Experimental and numerical demonstration of tunable octave-wide four-wave mixing in dispersion engineered microresonators (Invited Paper) [1 1274-19]

\section{PHOTODETECTION}

11274 OT Fundamental considerations for integrating silicon photomultipliers in frequency domain diffuse optical spectroscopy [11274-27]

11274 OU A Monte Carlo solution to hole transport processes in avalanche selenium semiconductors [1 $1274-28]$

11274 OX Prediction of optically triggered amplification in phototransistor with SPICE circuit simulators [1 1274-31]

11274 OY Gate-controlled time-dependent photocurrent in biologically inspired optoelectronics [1 $1274-37]$

$11274 \mathrm{OZ} \quad$ NIR optical properties of SWCNTs based on ab-initio calculations and the transfer matrix method [1 1274-32]

NONLINEAR PHOTONICS IN MICRO- AND NANOSTRUCTURES

1127412 Generating optical frequency combs via nanoscale photonic structures [1 1274-36]

PLASMONIC SENSING

1127414 Design and analysis of trench-based novel structure for high-sensitive surface plasmon resonance sensor [1 1274-39]

1127415 Surface plasmon resonance sensing structure [1 1274-40]

III-NITRIDE-BASED LASERS AND LEDS

1127416 Multi-scale modeling of electronic, optical, and transport properties of III-N alloys and heterostructures (Invited Paper) [1 1 $1274-41$ ]

$112741 \mathrm{~A}$ Investigation of intersubband phonon-polariton transitions in hBN/GaN heterostructure [1 1 274-45]

iv 
11274 1D A finite-difference time-domain formalism for second-order nonlinear effects [1 1274-48]

$11274 \mathrm{lE} \quad$ Opto-thermal simulation framework for the investigation of phosphor materials in laser-based lighting systems [1 $1274-49]$

\section{SEMICONDUCTOR LASERS AND NANO EMITTERS}

$1127411 \quad$ Rate equation analysis of digital pulse modulation in strongly injection-locked semiconductor microring lasers [1 $1274-53]$

$112741 \mathrm{~J} \quad$ Spectral linewidth narrowing of a quantum cascade laser by strong optical feedback [1 $1274-54]$

\section{INTEGRATED ULTRA LASERS}

$112741 \mathrm{~K} \quad$ High-performance mode-locked lasers on silicon (Invited Paper) [1 1274-55]

$112741 \mathrm{~L} \quad$ Hybrid integrated silicon nitride lasers (Invited Paper) [1 1274-56]

$112741 \mathrm{~N} \quad$ Monolithically integrated laser sources for applications beyond telecommunications (Invited Paper) [1 1274-58]

\section{POSTER SESSION}

$1127410 \quad$ Electron plasma parameter ferroelectric TGS [1 1274-59]

11274 IP Frequency characteristics of a semi-closed structure in a guided-wave optical pressure sensor for detection of tsunami formation: investigation based on numerical simulations and experiments [1 1274-60]

$112741 Q \quad$ Design of Si-photonics-based logic gates using micro-ring resonator structures [1 $1274-61]$

$112741 \mathrm{R} \quad$ Effect of corner radius on a single corner nanostructure for investigating SERS-based chemical sensors [1 $1274-62]$

11274 is Structural, electronic, and optical properties of type II heterostructure based on WS $2 /$ black phosphorene [1 1 1274-63]

$112741 \mathrm{~T} \quad$ Modelling and optimization of different plasmonic nanostructures for application in infrared region [1 1 274-64] 
$112741 \mathrm{U}$ Tuning the electronic and optical properties of graphene sheet by functionalization: an abinitio study [1 $1274-65]$

$11274 \mathrm{IV} \quad$ Wayfinding in complex buildings using visible light communication [1 1274-66]

11274 IW Femtosecond stimulated electro-optics of electrochemically synthesized CdS particles of different morphology [1 1274-67]

$112741 \mathrm{X}$ Laser-operated optical anisotropy of novel rare earth doped $\mathrm{TeO}_{2}-\mathrm{P}_{2} \mathrm{O}_{5}-\mathrm{ZnO}-\mathrm{M}_{\mathrm{x}} \mathrm{O}_{\mathbf{y}}-\mathrm{PbF}_{2}$ glasses [11274-68]

11274 1Y Carrier lifetime of black silicon as a photoconductor [1 1274-69]

$1127421 \quad$ An optical pulse generation technique using two optical phase modulators and a Fabry-Perot etalon [1 1274-72]

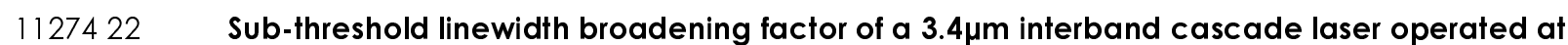
room temperature [1 $1274-73]$

$1127424 \quad$ Performance of infrared endoscope systems for laparoscopic surgery [11274-75]

1127426 Research of aberration control in human-eye inspired vari-focal liquid lens [11274-77]

1127429 Analysis of characteristics concerning volume phase gratings of which refractive index distributions are continuously changeable graded types and trapezoid types [1 1274-80]

112742 A A highly coherent multi-section semiconductor swept source OCT laser [1 1274-81]

$112742 B \quad$ Simulation of a parallel waveguide array structure suitable for interrogation scheme in a plasmonic biosensor [1 $1274-83]$

11274 2C Single-mode AIGaAs/InGaAs/GaAs lasers with a narrow waveguide [1 1274-84]

$112742 \mathrm{D} \quad$ Analysis of sensing properties of bending in optical fiber for highly sensitive applications [1 $1274-85]$

$112742 \mathrm{E} \quad$ High-aperture systems design for agriculture observation [1 1 274-86]

$112742 \mathrm{~F}$ Performance modeling of III-V antimonide-based barrier infrared detectors [1 1274-87]

$112742 \mathrm{~J} \quad$ Photoluminescence and time-resolved photoluminescence study of GaSe $\mathbf{~}_{1-\mathbf{x}} \mathbf{S}_{\mathbf{x}}$ mixed crystal [11274-91] 


\section{Authors}

Numbers in the index correspond to the last two digits of the seven-digit citation identifier (CID) article numbering system used in Proceedings of SPIE. The first five digits reflect the volume number. Base 36 numbering is employed for the last two digits and indicates the order of articles within the volume. Numbers start with 00, 01, 02, 03, 04, 05, 06, 07, 08, 09, OA, OB...0Z, followed by 10-1Z, 20-2Z, etc.

\begin{tabular}{|c|c|}
\hline A., Philippe, 08 & Francis, Henry, 12 \\
\hline Akis, Richard, OU & Fujii, Shun, OM \\
\hline Akkaya, Ibrahim, 21 & Gaafar, Mahmoud A., 04 \\
\hline Albassam, Ahmed A., IW & Gao, Fei, OA \\
\hline Alfano, Robert R., $1 \mathrm{Y}$ & Gavrina, Polina, OK \\
\hline Anand, Kartikeya, 2D & Geskus, Dimitri, $1 \mathrm{~L}$ \\
\hline Antonik, Piotr, OF & Geuzebroek, Douwe, $1 \mathrm{~L}$ \\
\hline Auth, Dominik, $1 \mathrm{~K}$ & Giudici, Massimo, OL, 2A \\
\hline Azhigulov, Dias, $1 Q$ & Goldan, A. H., OU \\
\hline Bai, Jing, 030 & Gomółka, E., 2F \\
\hline Beltako, K., OY & Gossard, Arthur, IK \\
\hline Bente, E. A. J. M., IN & Gosztola, David, 03 \\
\hline Bienstman, Peter, OD & Gowda, Uday, OL \\
\hline Bobe, Alexandra, 24 & Grigorenko, Konstantin, 2A \\
\hline Bobretsova, Yulia K., OK, 2C & Grillot, Frédéric, $1 \mathrm{~K}$ \\
\hline Boller, Klaus J., $1 \mathrm{~L}$ & Guldner, Y., 08 \\
\hline Bosak, Ondrej, $1 \mathrm{X}$ & Gumbs, Godfrey, OA \\
\hline Bowers, John, $1 \mathrm{~K}$ & Hantschmann, Constanze, OJ \\
\hline Brever, Stefan, $1 \mathrm{~K}$ & Harkhoe, Krishan, OD \\
\hline Brunner, Daniel, OF & Heideman, René G., 1L \\
\hline Camelin, Patrice, 2A & Ho, Ching-Hwa, 2J \\
\hline Carnio, B. N., 1D & Hopkinson, Mark, 12 \\
\hline Carosella, F., 08 & Huang, Danhong, OA \\
\hline Chakrabarti, Subhananda, IR, IS, IT, IU & Huyet, Guillaume, 0L, 2A \\
\hline Chao, Wen-Ching, 2J & Iurov, Andrii, OA \\
\hline Charola, Shreyas, 05 & Jang, Der-Jun, 2J \\
\hline Chatzizyrli, Elisavet, 1E & Jia, Wei, 03 \\
\hline Chaudhuri, D., 16 & Jin, Chao-Yuan, 12 \\
\hline Che, Kai-Jun, 12 & Jung, Daehwan, 1K \\
\hline Chen, Si, 12 & Katumba, Andrew, OD \\
\hline Cherepanov, D., OY & Kennedy, M.J., IK \\
\hline Chow, Weng, $1 \mathrm{~K}$ & Khalii, Diaa, $\mathrm{OZ}$ \\
\hline Christol, P., 08 & Kitsmiller, Vincent J., ОТ \\
\hline Costa, João, 15, 2B & Kityk, Iwan V., IW, IX \\
\hline Dawson, Jeremy, IA & Knigge, A., Ol \\
\hline Deng, Yu, 22 & Koester, J.-P., Ol \\
\hline de Vaulchier, L.-A., 08 & Kop'ev, Peter S., 2C \\
\hline Dhasarathan, Vigneswaran, 05 & Koprucki, T., 16 \\
\hline Dong, Bozhang, $1 \mathrm{~K}$ & Kopytko, M., $2 \mathrm{~F}$ \\
\hline Dongre, Suryansh, is & Kovalev, Anton, $\mathrm{OL}, 2 \mathrm{~A}$ \\
\hline Dumont, Mario, $1 \mathrm{~K}$ & Kozhina, Anastasiya D., 2E \\
\hline Eich, Manfred, 04 & Kracht, Dietmar, $1 \mathrm{E}$ \\
\hline Elezzabi, A. Y., 1D & Krizman, G., 08 \\
\hline El-Naggar, Ahmed M., IW & Kubliha, Marian, $1 \mathrm{X}$ \\
\hline Epping, Jörn P., 1L & Kumar, Abhishek, 1R, 1S, 1T, $1 \mathrm{U}$ \\
\hline Fantoni, Alessandro, 15, 1V, 2B & Kumar, Ajay, 14 \\
\hline Farrell, P., 16 & Kumar, Manish, 14, 2D \\
\hline Ferreira, R., 08 & Lachmayer, Roland, IE \\
\hline Florido, Emmanuel A., 2J & Ladumor, Mayurkumar, 05 \\
\hline
\end{tabular}


Latkowski, S., $1 \mathrm{~N}$

Lee, Joo Ho, 26

Lee, Jun Sik, 26

Lee, Meng-En, 2J

Leinse, Arne, $1 \mathrm{~L}$

Liu, Huiyun, OJ

Liu, Songtao, $1 \mathrm{~K}$

Liu, Zizhuo, OJ

Lourenço, Paulo, 15, 2B

Louro, Paula, 15, 1V

Lyutetskiy, Andrei V., 2C

Manyk, T., 2F

Marchuk, Oleg V., IW

Marconi, Mathias, OL, 2A

Marquardt, $0 ., 16$

Marsal, Nicolas, OF

Martyniuk, P., 2F

Michelini, F., OY

Möhrle, Martin, $1 \mathrm{~L}$

Moroz, Irina A., IW

Mukherjee, Atreyo, OU

Nadtochenko, V., OY

Nakajima, Kaoru, 29

Netesova, Nadezhda P., 10

Neumann, Jörg, 1E

Nikolaev, Dmitry N., 2C

Norman, Justin, $1 \mathrm{~K}$

O'Donovan, M., 16

O'Hearn, Catherine, 1A

Ohkawa, Masashi, IP

Oldenbeuving, Ruud M., IL

Ono, Hana, IP

Osiński, Marek, 11

O'Sullivan, Thomas D., OT

Ozga, Katarzyna, 1W, 1X

Pandey, Nivedita, 1R, 1S, 1T, IU

Park, Hyo-Hoon, $1 Q$

Patel, Shobhit K., 05

Patra, Saroj Kanta, 16, 2A

Penty, Richard V., OJ

Perez, J.-P., 08

Perez, Phoebe Nicole G., 2J

Petrov, Alexander Yu., 04

Pikhtin, Nikita A., 2C

Pimenov, Alexander, OL

Piwonski, Tomasz, 2A

Radziunas, M., Ol

Raghuwanshi, Sanjeev Kumar, 14, 2D

Reben, Manuela, $1 \mathrm{X}$

Rebrova, Natalia, 2A

Renner, Hagen, 04

Roberts, Kevin, 03

Roche, Amy, OL, 2A

Rodriguez, J. B., 08

Roeloffzen, Chris G. H., $1 \mathrm{~L}$

Rontani, Damien, OF

Rossi, Chiara, OX

Roy, Aritra, 2A

Rudova, Natalia, OK

Rutkowski, J., 2F
Sabry, Yasser M., OZ

Saeed, Ahmed, OZ

Sallese, Jean-Michel, OX

Schell, Martin, $1 \mathrm{~L}$

Schulz, S., 16

Seeds, Alwyn J., 0J

Shamakhov, Viktor V., 2C

Shang, Chen, $1 \mathrm{~K}$

Shashkin, llya S., 2C

Shawkey, Heba A., OZ

Slepneva, Svetlana, OL, 2A

Slipchenko, Sergey O., OK, 2C

Smolyakov, Gennady A., 11

Soboleva, Olga, OK

Streckenbach, T., 16

Takaoka, Naoto, $1 \mathrm{P}$

Tanabe, Takasumi, OM

Tang, Mingchu C., OJ

Theurer, Michael, $1 \mathrm{~L}$

Tozburun, Serhat, 21

Triyama, Taiju, IP

Tsyganok, Helen A., 2E

Tu, Li-Wei, 2J

Ukaegbu, Ikechi A., 1Q

Van der Sande, Guy, OD

van Rees, Albert, $1 \mathrm{~L}$

Vasileska, Dragica, oU

Verschaffelt, Guy, OD

Verschelde, Alexis, 2A

Veselov, Dmitrii A., 2C

Vieira, M. A., IV

Vieira, Manuela, 15, 1V, 2B

Vieira, P., IV

Viktorov, Evgeny, OL, 2A

Visscher, llka, $1 \mathrm{~L}$

Vladimirov, Andrei, OL

Voznesenskaya, Anna, 24

Wan, Yating, $1 \mathrm{~K}$

Wang, Cheng, 1J, 22

Wang, Xing-Guang, $1 \mathrm{~J}$

Wenzel, $\mathrm{H}$., $\mathrm{Ol}$

White, lan H., OJ

Wiederrecht, Gary, 03

Wienke, Andreas, $1 \mathrm{E}$

Williams, K. A., $1 \mathrm{~N}$

Won, Yong Hyub, 26

Wu, Xinru, 1K

Yanchuk, Oleksandr M., IW

Yousef, El Sayed, $1 \mathrm{X}$

Zeghuzi, A., Ol

Zhang, Shengkun, $1 Y$

Zhang, Xiao-Dong, 12

Zhao, Bin-bin, $1 \mathrm{~J}$

Zhou, Debao, 03 


\section{Conference Committee}

Symposium Chairs

Sailing He, KTH Royal Institute of Technology (Sweden) and Zhejiang University (China)

Yasuhiro Koike, Keio University (Japan)

Symposium Co-chairs

Connie J. Chang-Hasnaian, University of California, Berkeley (United States)

Graham T. Reed, Optoelectronics Research Centre, University of Southampton (United Kingdom)

Program Track Chairs

James G. Grote, Photonics Engineering Consultant (United States)

Shibin Jiang, AdValue Photonics, Inc. (United States)

Conference Chairs

Bernd Witzigmann, Universität Kassel (Germany)

Marek Osiński, The University of New Mexico (United States)

Yasuhiko Arakawa, The University of Tokyo (Japan)

Conference Program Committee

Hiroshi Amano, Nagoya University (Japan)

Toshihiko Baba, Yokohama National University (Japan)

Jing Bai, University of Minnesota, Duluth (United States)

Enrico Bellotti, Boston University (United States)

Guillermo Carpintero, Universidad Carlos III de Madrid (Spain)

Weng W. Chow, Sandia National Labs. (United States)

Alexandre Freundlich, University of Houston (United States)

Michael D. Gerhold, U.S. Army Research Office (United States)

Frédéric Grillot, Télécom ParisTech (France)

Ortwin Hess, Imperial College London (United Kingdom)

Stephan W. Koch, Philipps Universität Marburg (Germany)

Kathy Lüdge, Technische Universität Berlin (Germany)

Cun-Zheng Ning, Arizona State University (United States)

Joachim Piprek, NUSOD Institute LLC (United States)

Marc Sciamanna, CentraleSupélec (France) 
Ikuo Suemune, Hokkaido University (Japan)

Kaikai Xu, University of Electronic Science and Technology of China (China)

\section{Session Chairs}

1 Electromagnetics/Plasmonics I

Bryan Kelleher, University College Cork (Ireland)

2 Active Materials for Optoelectronics

Stefan Schulz, Tyndall National Institute (Ireland)

3 Minisymposium on Neuromorphic Computing with Optical Devices

Bernd Witzigmann, Universität Kassel (Germany)

4 Semiconductor Lasers: Numerical Modeling

Takasumi Tanabe, Keio University (Japan)

$5 \quad$ Signal Encryption and Nonlinear Optical Systems

Volker J. Sorger, The George Washington University (United States)

6 Quantum Dot Emitters

Thorsten S. Rasmussen, Technical University of Denmark (Denmark)

Jesper Mørk, Technical University of Denmark (Denmark)

7 Photodetection

Dimitris Syvridis, National and Kapodistrian University of Athens (Greece)

8 Nonlinear Photonics in Micro- and Nanostructures

David De Felipe Mesquida, Fraunhofer-Institut für Nachrichtentechnik, Heinrich-Hertz-Institut, HHI (Germany)

9 Plasmonic Sensing

Jia-Gui Wu, University of California, Los Angeles (United States)

10 III-Nitride based Lasers and LEDs

Emmanouil Kioupakis, University of Michigan (United States)

11 Electromagnetics/Plasmonics II

Gergo P. Szakmany, University of Notre Dame (United States)

12 Semiconductor Lasers and Nano Emitters

Satoshi Iwamoto, The University of Tokyo (Japan)

13 Integrated Ultra Lasers

Guillermo Carpintero, Universidad Carlos III de Madrid (Spain) 